\title{
Driving issues of large area liquid crystal devices
}

\author{
Boxuan $\mathrm{Gao}^{\mathrm{a}}$, Ties de Jong ${ }^{\mathrm{b}}$, Chuan $\mathrm{Nie}^{\mathrm{b}}$, Jens Osterodt ${ }^{\mathrm{b}}$, Kristiaan Neyts ${ }^{\mathrm{a}}$ and \\ Jeroen Beeckman ${ }^{\mathrm{a}}$ \\ ${ }^{a}$ Electronics and Information Systems Department, Ghent University, Ghent, Belgium; \\ b Merck Window Technologies, Veldhoven, The Netherlands
}

\author{
ARTICLE HISTORY \\ Compiled July 9, 2020
}

\begin{abstract}
The surface area of liquid crystal (LC) devices in different applications, such as displays, signage devices and smart windows is becoming larger and larger. The electrical driving of such devices does not pose many problems when working on centimetre sized devices. Different types of driving issues arise when up-scaling such devices to areas of several square meter, due to the fact that the thin-film transparent conductors used in these device exhibit a finite sheet conductivity. One of the resulting issues is the non-uniformity of the electric field across the area of the device and the resulting non-uniform optical behaviour. In this work we present a simulation model that is able to predict the optical and electrical behaviour of large-sized liquid crystal devices with different applied voltage signals, including the power consumption. The simulation results are compared with optical and electrical measurements on a liquid crystal device of $1.5 \mathrm{~m}$ by $1.2 \mathrm{~m}$.
\end{abstract}

\section{KEYWORDS}

Nematic liquid crystals; optical properties; electric measurements; energy use and applications; numerical methods

\section{Introduction}

Liquid crystal devices are widely present in our daily lives. The size of such devices ranges from about one centimetre, as in Liquid Crystal on Silicon (LCOS) devices for projection systems, to several meter in the latest generation of television sets. One other application of liquid crystals in which sizes of several meters are desired are smart windows. Smart windows that switch between an opaque and a transparent state in response to external stimuli have a wide potential for applications, for example in energy efficient buildings [1-3]. Blocking the sunlight can reduce the cost for air conditioning in the summer, while transmitting sunlight during the winter reduces heating requirements. Another application for smart windows could also be in Augmented Reality (AR)[4], where the window is electrically switched between clear and scattered state. Different technologies to realize smart windows exist, ranging from chromic materials [5-7] such as electrochromic devices [8], liquid crystal devices [9] to electrophoretic or suspended-particle devices $[1,10]$.

In the case of LC based smart windows, the switching mechanism is based on the reorientation of the average LC orientation (i.e. the director) using an external electric

Email: jeroen.beeckman@ugent.be 



Figure 1. Liquid Crystal Smart Window operation. (a) Smart window switches from the opaque state to the transparent state in response to the applied voltage. (b) Smart window switches from low-transmission to high-transmission in response to the applied voltage.

field $[11,12]$. The change in orientation will lead to changes in the light transmission, as indicated in fig.1. Depending on how the light is transmitted in the two states of the window, it can either function as a privacy window or as an energy saving window $[11,13,14]$. In privacy windows [15] (fig.1(a)), the light is highly scattered in one state such that objects behind the window are not visible. The scattering may be caused for example by the random orientation of LC droplets in Polymer Dispersed Liquid Crystal (PDLC) devices [16,17], Polymer-Stabilized LC (PSLC) devices [18] or by a focal conic state in chiral LC devices [19]. When there is an external voltage applied, the director will align along the electric field leading to a uniform refractive index in the layer and a transparent state without scattering. In energy saving windows (fig.1(b)), the amount of light transmitted through the window changes drastically between the different states of the window. A common way to change the transmission without using polarizers is based on the guest-host principle, using a dichroic absorbing dye (the guest) dissolved in a liquid crystal (the host), that aligns along the director [20].

In electrically controllable liquid crystal smart windows, the liquid crystal layer is held between two glass plates, which are coated with a transparent conductive layer. The thickness of this conductive layer is chosen in order to reach an optimal balance between high light transmission and high sheet conductivity [21]. Indium tin oxide is widely used as transparent conductive layer. However, other types of transparent conductors have also been studied such as silver nanowires or graphene [17,22,23]. Although the liquid crystal material itself is quasi non-conductive, the presence of small amounts of ions requires driving with Alternating Current (AC). In the case of a static voltage applied, the positive (negative) ions would rapidly move towards the negative (positive) electrode and screen the applied electric field $[24,25]$. The use of AC voltages to drive the smart window automatically leads to currents in the electrodes that lead to an interplay between the resistance of the transparent electrodes and the capacitance of the liquid crystal layer. Above a certain frequency, it is clear that the associated RC-effect will become important and lead to an important voltage drop over the area of the transparent electrode. This lateral voltage drop leads to a reduction in the effective voltage over the liquid crystal layer. It is this effective voltage that determines the liquid crystal director distribution over the cell thickness, as well as the transmission.

The effective voltage over the LC layer will be non-uniform over the device area due to the RC-effect, especially in large-size devices that are driven at a high frequency. This distribution will depend on the geometry of the device, the contacting scheme, the LC layer thickness, the LC permittivity and its anisotropy. Quantifying this effect analytically is possible for a number of simplified geometries, as demonstrated in [26], 
but for more complex geometries, numerical simulations are necessary to evaluate the voltage and transmission distribution.

Non-uniformity effect due to finite conductivity of the transparent conductors occurs in a wide variety of devices, and often the non-uniformity is related to purely resistive effects. In large area Organic Light Emitting Diodes (OLEDs) for lighting applications a drop in light emission is observed due to the finite sheet conductivity. An intelligent design of the conductor grids may help to reduce lateral variations $[27,28]$. Similar effects are playing a role in solar cells in which the generated current needs to be collected by the electrode grid in an efficient way [29]. In all these cases it is important to have a numerical model to estimate resistive losses.

In this work, one large-sized LC device is numerically simulated and experimentally studied to investigate the effect of the variable voltage drop over the area. The device consists of two ITO coated glass plates with an alignment layer that is anti-parallel rubbed and is filled with Merck's LC mixture E7. External voltage is applied through the contacts placed on the edges of the ITO layer, and distributed over the surface. When there is an external voltage applied, the liquid crystal aligns along the normal to the substrates, as indicated in fig.1(b). To facilitate the estimation of the voltage drop from experiments, no dichroic dye is added to the liquid crystal. To evaluate the director orientation, the spectral transmission of the device between two crossed polarizers is measured experimentally and transferred into the corresponding voltage values for the comparison with the simulation.

\section{Method}

In this study, the theoretical numerical model that was worked out in reference [26] is adapted to simulate RC-effects in the up-scaled liquid crystal cell, wherein the behaviour of a nematic liquid crystal between two substrates with finite sheet conductivity is simulated. For this goal, two sets of differential equations have to be implemented. One set of equations describes the potential distribution in the conductive layers, considering the coupling between the resistivity of the conductive layers and the local capacitance of the LC layer. The other set describes the liquid crystal director distribution and the resulting capacitance according to the effective voltage over the layer. The thickness of the LC layer is much smaller than the height and width of the LC cell and therefore the LC orientation can be solved locally with a one-dimensional differential equation. Since the two sets of differential equations are coupled, an iterative scheme is implemented to obtain the effective voltage distribution (and spectral transmission) as a function of position across the area of this device.

The position dependent effective voltage $\left|V_{1}-V_{2}\right|$ across the liquid crystal layer is found by simulating the potential distributions $V_{1}(x, y)$ and $V_{2}(x, y)$ respectively at the top and the bottom conductive layers, with $x$ and $y$ the horizontal and vertical coordinate, which determine the position on the cell. As we work in the frequency domain, the two voltages are complex, representing phasors. A two-dimensional finite element solver is used to find a solution for the following two coupled differential equations:

$$
\nabla_{\perp}^{2} V_{1}=\frac{j \omega \varepsilon_{\mathrm{eff}}}{\sigma_{1} d_{1} d}\left(V_{1}-V_{2}\right)
$$




$$
\nabla_{\perp}^{2} V_{2}=\frac{j \omega \varepsilon_{\text {eff }}}{\sigma_{2} d_{2} d}\left(V_{2}-V_{1}\right)
$$

This set of equations, expressing that the capacitive charging on the LC is supplied by lateral currents in the electrodes, has been derived in detail in reference [26]. Note that the Laplacian contains only the $x$ and $y$ components. In this equations $\omega$ is the angular frequency of the applied voltage. The product of the conductivity $\sigma_{1}\left(\sigma_{2}\right)$ and the thickness of the weakly conductive layer $d_{1}\left(d_{2}\right)$ denotes the sheet resistance, $\varepsilon_{\text {eff }}(x, y)$ is the position-dependent effective permittivity of the LC layer. As the lateral dimensions of the window are much larger than the thickness $d$ of the LC layer, the effective permittivity can be calculated by noting that the $z$-component of the dielectric displacement field is a constant. Expressing this leads to the following equation for calculating the effective dielectric constant (with $\varepsilon_{z z}$ the component of the permittivity tensor along $z$ ):

$$
\epsilon_{\mathrm{eff}}=\frac{d}{\int_{0}^{d} \frac{1}{\epsilon_{z z}(z)} d z}
$$

As the liquid crystal orientation is determined by the applied voltage over the layer, the effective permittivity is a function of the applied voltage: $\varepsilon_{\text {eff }}\left(\left|V_{1}-V_{2}\right|\right)$. In the frequency domain formalism, the potentials $V_{1}$ and $V_{2}$ are complex, but the liquid crystal response depends only on the absolute value of the potential difference. The exact relation between $\varepsilon_{\text {eff }}$ and $\left|V_{1}-V_{2}\right|$ depends on the parameters of the liquid crystal layer, as will be discussed later. Important to note is that the coupled differential equations would be linear if $\varepsilon_{\text {eff }}$ were a constant (i.e. independent of the applied voltage). However, the differential equations are generally non-linear. An iterative method is used to obtain the final voltage distribution from formulas (1) and (2). Since $\varepsilon_{\text {eff }}$ is typically a smooth function of applied voltage, only a limited number of iterations are necessary until sufficient accuracy is obtained. In this simulation, the accurate voltage distribution is considered to be achieved when the relative change is smaller than $10^{-6}$.

With this model, the behaviour of a large-sized liquid crystal window is simulated. The liquid crystal is the mixture E7 (Merck), which is aligned parallel to the substrate surface with a pretilt of two degrees. The in-plane alignment directions at the two substrates are anti-parallel. When a voltage is applied, the liquid crystal director will align towards the direction of the electric field, which has a mutual influence on the local voltage due to the RC-effect. The calculation of this orientation distribution along $z$ as a function of voltage is based on the minimization of the total (elastic and electric) energy, using strong anchoring conditions. For details about the governing equations and solution methods we refer to the literature available on this topic [30,31]. The tilt angle $\theta$ along the thickness of the LC layer is shown in fig.2(a) for three different voltages. This distribution determines the effective permittivity of the liquid crystal layer and the simulated $\varepsilon_{\text {eff }}$ as a function of applied voltage is shown in fig.2(b), where the relative permittivity used for this simulation is 19.6 and 5.1 for the parallel and perpendicular case respectively. As the liquid crystal reorients, the refractive index experienced by the incident light changes. To observe this through the transmission change, the LC cell is sandwiched between two crossed polarizers, with an angle of $45^{\circ}$ with respect to the fast axis of the liquid crystal, as shown in fig.3(d). Light travelling through the LC layer sees a retardation proportional to $\Delta n d$ where 
(a)


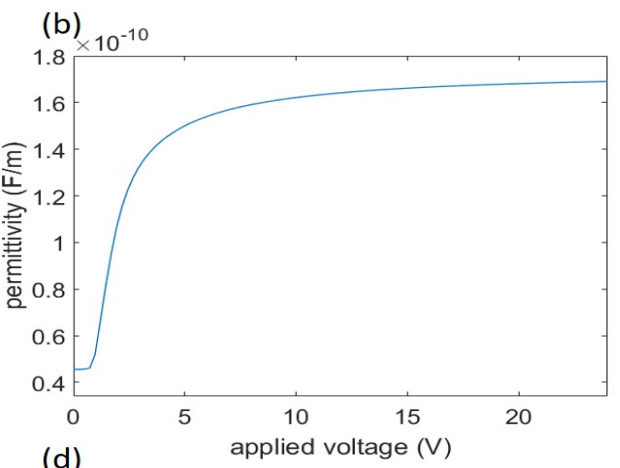

(d) applied voltage (V)

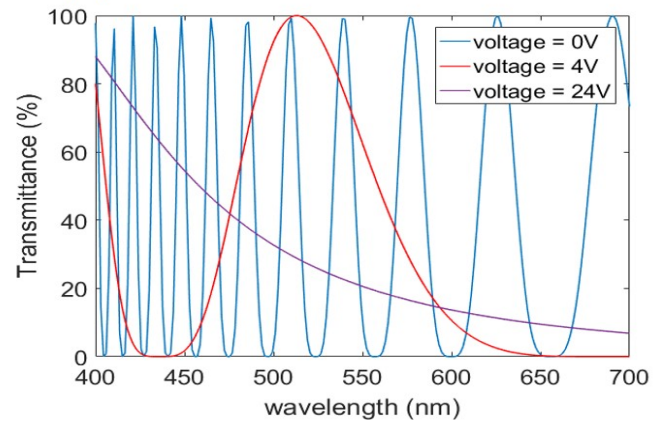

Figure 2. (a) The tilt angle of the LC orientation along the direction perpendicular to the cell surface for different voltages. (b) Effective permittivity of the liquid crystal, as a function of applied voltage. (c) Phase retardation experienced by light passing through a LC layer with thickness $25 \mu \mathrm{m}$, as a function of applied voltage. (d) Transmission spectra for three different applied voltages.

$$
\Delta n d=\int_{0}^{d}\left(n_{e f f}(z)-n_{\perp}(z)\right) d z
$$

The total retardation $\Delta n d$ of the LC layer is calculated by integrating the local $\Delta n$ over the LC layer thickness. According to this retardation, part of the light with a certain wavelength will pass through the second polarizer. As the liquid crystal reorientation is subject to the applied voltage, this total retardation also varies as a function of voltage, as shown in fig.2(c). The transmission spectrum for three different applied voltages is shown in fig.2(d), based on the Jones matrix formalism, taking into account the dispersion of the refractive index of E7 as found in ref.[32], as shown in eq.5 and 6.

$$
\begin{aligned}
& n_{e}(\lambda)=1.6933+\frac{0.0078}{\lambda^{2}}+\frac{0.0028}{\lambda^{4}} \\
& n_{o}(\lambda)=1.4990+\frac{0.0072}{\lambda^{2}}+\frac{0.0003}{\lambda^{4}}
\end{aligned}
$$




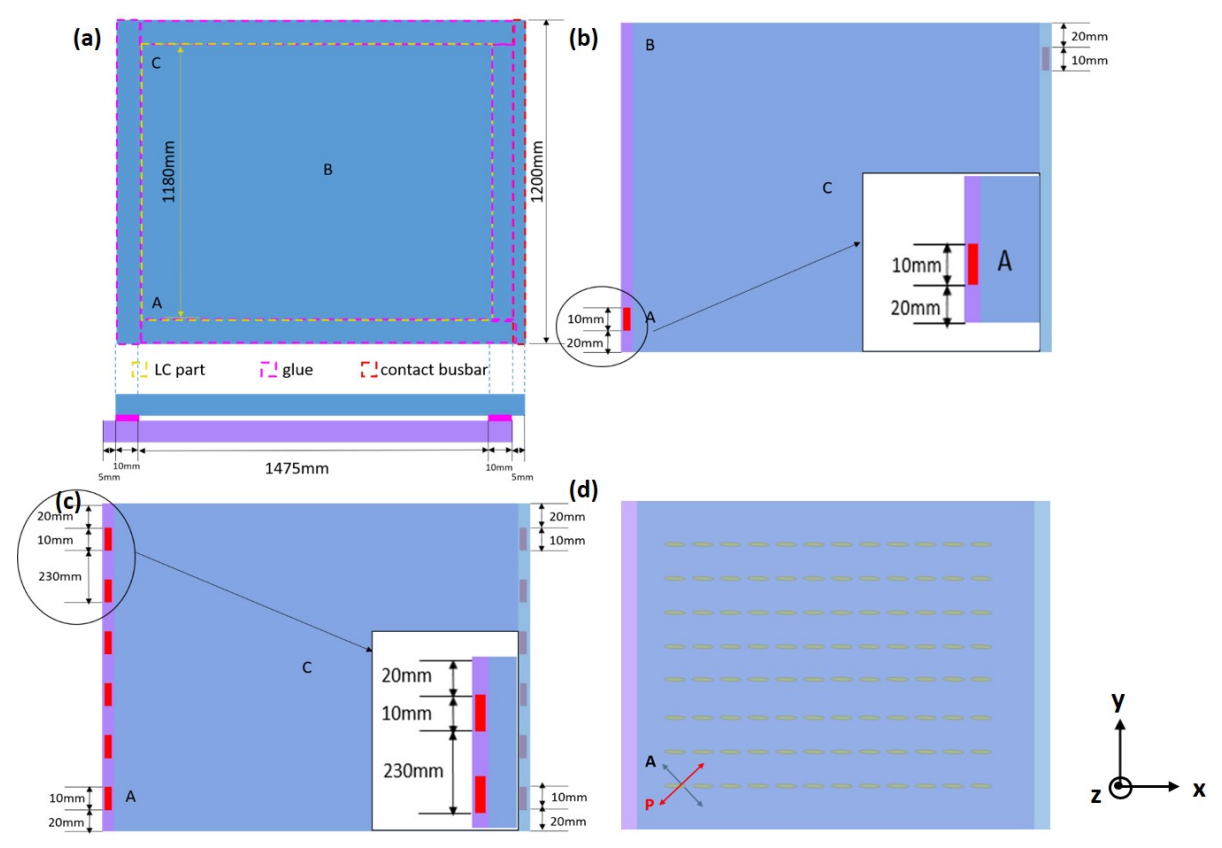

Figure 3. (a) Geometry of the up-scaled LC cell used in this work. The top figure is the top view with coloured dashed lines showing different regions. The bottom one is the side view, the magenta part shows the glue. (b) and (c) show single contact and dashed line contact busbar formats respectively. A, B and C indicate the experimental measured points. The insets of these three figures indicate the dimensions and formats of the busbar. (d) Schematic view of the direction of the two crossed polarizers (P: polarizer. A: analyser) and the liquid crystal fast axis. There is an angle of $45^{\circ}$ between the fast axis and each polarizer.

\section{Sinusoidal frequency domain numerical simulation and experiment}

The LC model used in this work has two substrates of $1500 \mathrm{~mm} \times 1200 \mathrm{~mm}$, with further geometrical details outlined in fig.3(a). At the left and right side, the bottom and top glass plates are not overlapping in order to have space for electrical contacts (the busbars). Part of the substrate is covered with glue for making a LC cell. As a result, the effective area of overlapping ITO electrodes with liquid crystal in between is $1475 \mathrm{~mm} \times 1180 \mathrm{~mm}$. The contact busbar regions at the left and the right side have dimensions $5 \mathrm{~mm} \times 1200 \mathrm{~mm}$. The sheet resistance for the conductive layer is set to be $100 \Omega / s q$. In this work, two different busbar formats have been tested, namely single contact and dashed line contact. The busbars are soldered contacts and in the simulations, these regions are treated as perfect conductors. The spectral transmission through the device is measured at a number of locations. Since the transmission is varied for different wavelengths, the corresponding voltage that makes the transmission match for all wavelengths is extracted from the experimentally measured transmission spectrum, and compared with the simulation results.

\subsection{Contact format: single contact}

For the single contact format, two rectangular contacts with length around $10 \mathrm{~mm}$ are put on the opposite two corners of the sample in the busbar region, on each of the substrate respectively, as shown in fig.3(b), $20 \mathrm{~mm}$ away from the up and bottom edge respectively. The applied voltage is a sinusoidal wave with root mean square (rms) value of $4 \mathrm{~V}$ or $24 \mathrm{~V}$ and a frequency of $60 \mathrm{~Hz}$ or $600 \mathrm{~Hz}$, resulting in a total of four 



Figure 4. Voltage distribution calculated for applied sinusoidal signal with voltage $4 \mathrm{~V}$ and frequency $60 \mathrm{~Hz}$. (a) Top conductive layer connected with $4 \mathrm{~V}$. (b) Bottom conductive layer connected with $0 \mathrm{~V}$.

different simulation conditions that will be discussed below.

In the first simulation, the sinusoidal wave with rms $4 \mathrm{~V}$ and frequency $60 \mathrm{~Hz}$ is applied. The absolute value of the potential distributions $V_{1}(x, y)$ (top conductive layer connected with voltage $4 \mathrm{~V}$ ) and $V_{2}(x, y)$ (bottom layer connected with voltage $0 \mathrm{~V}$ ) are plotted in fig.4. As explained above, the liquid crystal orientation, thus as well as the effective permittivity and the retardation, is dependent on $\left|V_{1}-V_{2}\right|$. Therefore the effective voltage distribution over the entire liquid crystal layer is obtained by taking the absolute difference between the voltages on the top and bottom layer, as shown in fig.5(a). Because of the capacitance of the LC layer, the applied voltage experiences a drop over the cell according to the local orientation of the LC, which is in turn influenced by the applied voltage. Higher applied voltage leads to stronger RC effect (due to a higher capacitance) and thus a larger voltage drop. As a result of the voltage drop the RC effect becomes weaker and finally achieves a balance. For a large-scale device, it is essential to find out how this RC effect disturbs the homogeneity across the device, which in this paper will be described by the voltage distribution. The highest voltage is found next to the contact, with a value of $2.60 \mathrm{~V}$. Note that even close to the contact, there is a considerable decrease of voltage compared to the applied voltage of $4 \mathrm{~V}$. In a small region around the single contact, the voltage gradually drops and eventually reaches a minimum of $2.06 \mathrm{~V}$ at the centre of the device. Around the centre of the device, the voltage is fairly homogeneous. It is also possible to simulate the transmission for the whole cell based on the obtained voltage distribution. However, this type of $\mathrm{LC}$ cell is not used in real applications as the transmission varies strongly with wavelength, as shown in fig.2. The E7 mixture is used here as its properties are widely known and accurate results can be extracted from the E7 cell. To give the reader an idea of the effect in practical devices, the transmission distribution of a dye doped device will be presented later. Also, it should be mentioned that the voltage drop going from the corner to the middle is relatively small (about $0.5 \mathrm{~V}$ ) compared to the driving voltage of $4 \mathrm{~V}$ (a drop of 12.5\%). It is therefore important in the analysis and discussion of further simulation examples that not only the homogeneity in switching is considered, but also the voltage drop compared with the driving voltage.

As the frequency increases to $600 \mathrm{~Hz}$, the voltage across the device decreases in general due to the fact that the $\mathrm{RC}$ effect becomes stronger. The maximum voltage of $1.91 \mathrm{~V}$ is found around the contact corners and the voltage drops to a minimum value of $0.58 \mathrm{~V}$, corresponding with a $33.25 \%$ voltage drop with respect to the driving voltage. Although the voltage drops more under a higher driving frequency, the voltage 

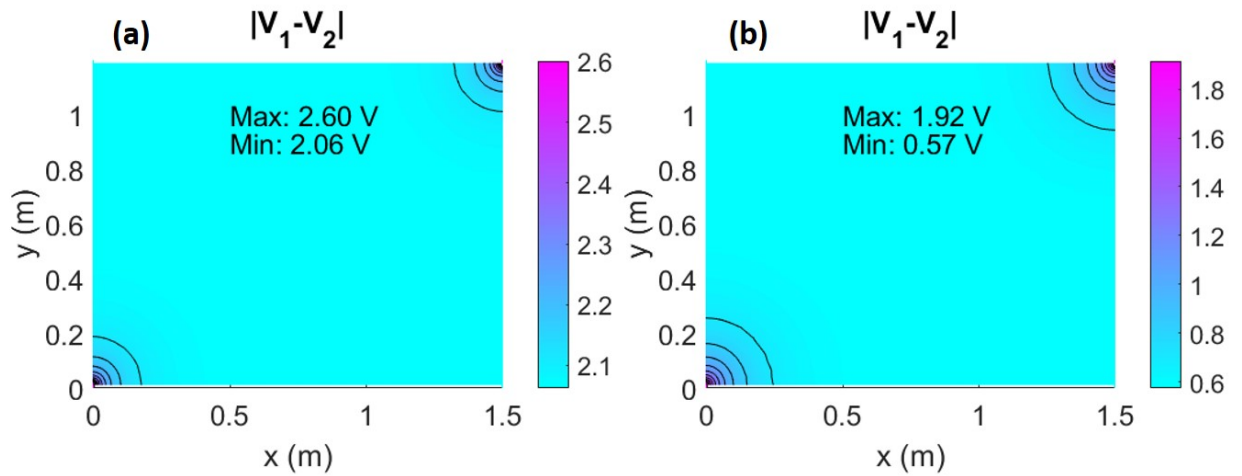

Figure 5. Simulation results for applied sinusoidal signal with voltage 4 V. (a) Voltage distribution with applied frequency $60 \mathrm{~Hz}$. (b) Voltage distribution with applied frequency $600 \mathrm{~Hz}$.

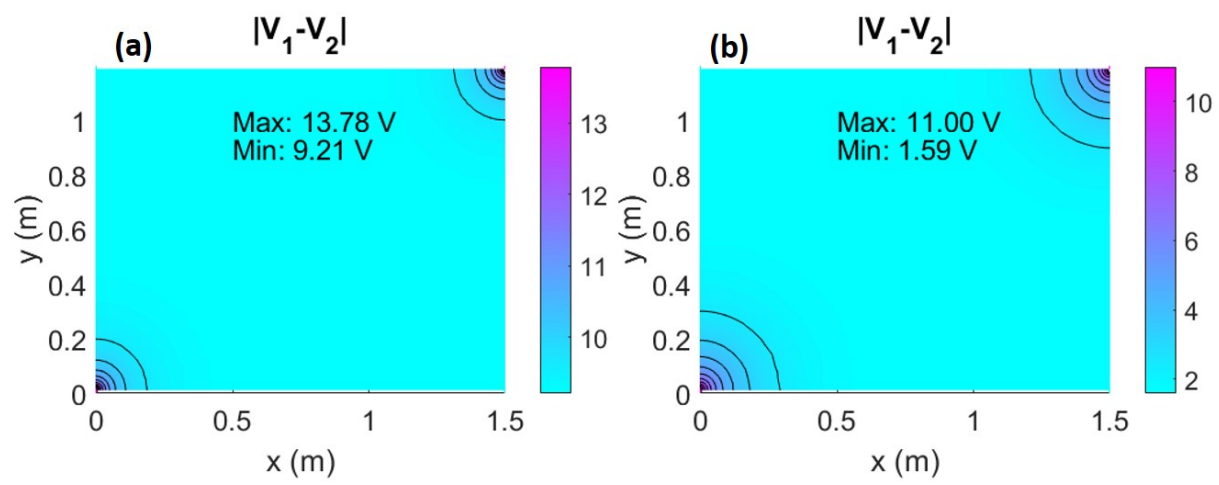

Figure 6. Simulation results for applied sinusoidal signal with voltage $24 \mathrm{~V}$. (a) Voltage distribution with applied frequency $60 \mathrm{~Hz}$. (b) Voltage distribution with applied frequency $600 \mathrm{~Hz}$. 
distribution across the cell is still fairly uniform.

Also for a driving voltage of $24 \mathrm{~V}$, two frequencies are simulated. One might imagine that the results for higher voltages can be extrapolated from the $4 \mathrm{~V}$ case, but this is not true because the liquid crystal responds nonlinearly to the voltage and the capacitance of the liquid crystal layer is changing with voltage. The capacitance increases for higher voltages, so stronger RC effects are expected for higher voltages. The simulation result for a frequency of $60 \mathrm{~Hz}$ is shown in fig.6(a). The maximum voltage near the contacts reaches a value of $13.78 \mathrm{~V}$, and eventually drops to a minimum voltage of $9.21 \mathrm{~V}$. This results in a relative voltage drop compared with the driven voltage of $19.04 \%$. The percentual drop in the voltage of $19.04 \%$ is clearly larger than the value for $4 \mathrm{~V}$ driving voltage (being 12.5\%). Again, although there is a considerable voltage drop across the device, the voltage distribution is still fairly uniform in the central part.

Finally, the simulation for the high frequency $600 \mathrm{~Hz}$ is also shown in fig.6(b). With the maximum voltage of $11.00 \mathrm{~V}$ near the contacts and a minimum $1.59 \mathrm{~V}$, the voltage drop across the cell is $39.21 \%$ compared to the driving voltage. The large drop is expected due to the high voltage (higher capacitance of LC layer) and the high frequency (stronger RC-effect in general).

To have an idea how the transmission would be influenced in a dye-doped LC cell, transmission simulations are conducted for a LC-dye mixture with dichroic ratio of 10 . The dichroic dye concentration is such that the absorption coefficients for parallel and perpendicular polarization direction are 0.04 per $\mu \mathrm{m}$ and 0.004 per $\mu \mathrm{m}$ respectively. The wavelength chosen for the simulation is $500 \mathrm{~nm}$. When there is no voltage applied, the effective absorption efficient is 0.04 per $\mu \mathrm{m}$ with the corresponding transmission of $34.44 \%$. As the LC reorients according to the effective voltage distribution, the dye will reorient along with the LC, thus the light passing through the mixture layer will experience a different absorption based on the orientation. When the external voltage applied is $4 \mathrm{~V}, 60 \mathrm{~Hz}$ and $24 \mathrm{~V}, 60 \mathrm{~Hz}$ respectively, the corresponding transmission distribution is shown in fig.7. With higher voltage, the transmission is higher, because the perpendicular absorption is weaker. Same as the voltage distribution, there are some drops at the corner where the contacts locate, but in general the distribution is rather homogeneous. The uniformity of the light transmission can be further improved using a dashed line contact format, as will be demonstrated later in the voltage distribution simulation, or even better with a complete line contact. Still, for a large-sized device with dimension $1500 \mathrm{~mm} \times 1200 \mathrm{~mm}$ and only two contact dots, the transmission uniformity is already acceptable. For an applied voltage of $4 \mathrm{~V}$, the transmission drop at the corner is around $4 \%$ and for applied voltage $24 \mathrm{~V}$ it is around $2 \%$, which will have minor influence on the cell behaviour. Since the contact soldering is costly in terms of time and material use, for every situation, a balance must be found between transmission uniformity and amount of contacts.

According to the previously simulated voltage distribution, the power consumption of the large-sized LC cell can be calculated from the simulation data. The following formula expresses the power consumption, being a line integral circumventing the contacts.

$$
P=\sum_{i=1,2} \oint_{L} \bar{K}_{i}^{*} V_{i} \cdot\left(\overline{1}_{z} \times d \bar{l}\right)
$$


(a)



(b)

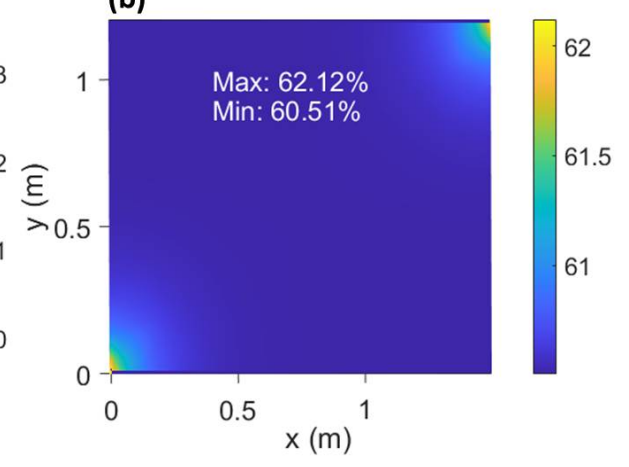

Figure 7. Simulation for transmission through the guest-host cell. (a) Applied voltage is $4 \mathrm{~V}, 60 \mathrm{HZ}$. (b) Applied voltage $24 \mathrm{~V}, 60 \mathrm{~Hz}$. The transmission for zero applied voltage is: $34.44 \%$.

Table 1. Power consumption simulated for single contact format

\begin{tabular}{lllll}
\hline voltage format & $4 \mathrm{~V} 60 \mathrm{~Hz}$ & $4 \mathrm{~V} 600 \mathrm{~Hz}$ & $24 \mathrm{~V} 60 \mathrm{~Hz}$ & $24 \mathrm{~V} 600 \mathrm{~Hz}$ \\
\hline power consumption $(\mathrm{W})$ & $0.019+\mathrm{j} 0.0119$ & $0.0254+\mathrm{j} 0.0042$ & $0.7949+\mathrm{j} 0.3450$ & $0.9418+\mathrm{j} 0.0955$ \\
\hline ideal power consumption $(\mathrm{W})$ & $\mathrm{j} 0.0622$ & $\mathrm{j} 0.622$ & $\mathrm{j} 2.2393$ & $\mathrm{j} 22.393$ \\
\hline
\end{tabular}

In this equation $\bar{K}_{i}$ is the surface current in the top or bottom sheet conductor given by $\bar{K}_{i}=\sigma_{i} d_{i} \nabla_{\perp} V_{i}$ with $i=1,2$. Note that in this equation for the power consumption, there is a real part (the resistive losses from the transparent conductor) and an imaginary part (capacitive losses from the LC layer). From this equation the power is calculated as shown in table 1 .

To verify the simulation results, measurements are conducted by recording the spectral transmission of the cell at different locations. As in the simulation, the glass substrate used for the cell has a size of $1.5 \mathrm{~m} \times 1.2 \mathrm{~m}$ and the liquid crystal used is the E7 mixture, which are the uniaxial nematic liquid crystals. The whole LC cell is fabricated to have a $25 \mu \mathrm{m}$ thick liquid crystal layer and the coated ITO conductive layer has a sheet resistance of $\sim 100 \Omega / s q$.

To probe the orientation of the liquid crystal for different applied voltages using the spectral transmission, the device is placed between two crossed polarizers with an angle of $45^{\circ}$ to the LC director, as shown in fig.3(d). The polarization direction of the incident light on the LC cell will have a $45^{\circ}$ angle with the projection of the director onto the $x y$-plane. As the light is propagating through the cell, the polarization component along and perpendicular to the projection of the director onto the $x y$-plane will experience different refractive indices. As a result, the polarization state of the light will change. Due to the presence of the second polarizer, the light transmission will vary as a function of wavelength according to the following equation:

$$
T=T_{0} \sin ^{2} \frac{\pi \Delta n_{\mathrm{eff}} d}{\lambda}
$$

The resulting wavelength dependent transmission is shown in fig.2(d). When there is an externally applied electric field, the liquid crystal tends to reorient to be perpendicular to the substrates. With increasing voltage the effective retardation $\Delta n_{\text {eff }} d$ decreases as shown in fig.2(c), leading to transmission spectra with less wavelength variation. From the transmission spectrum, the corresponding voltage can be extracted through a Matlab program and compared with the simulation results. 

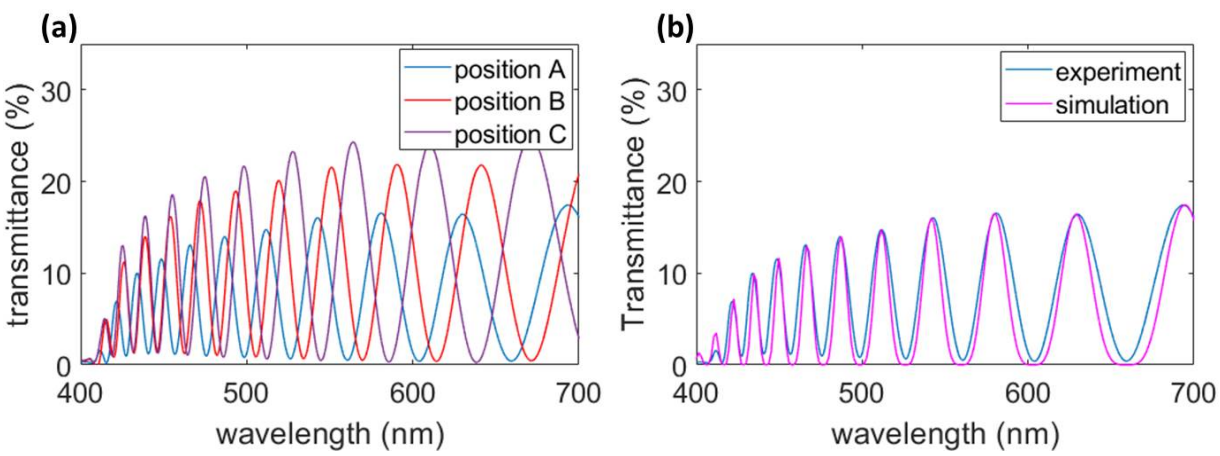

Figure 8. (a)Transmission spectra of position A, B and C, when there is no voltage applied. (b)Transmission spectra of position $\mathrm{A}$, for both experiment and simulation.

Table 2. Comparison of the voltage between simulation and experiment for single contact format

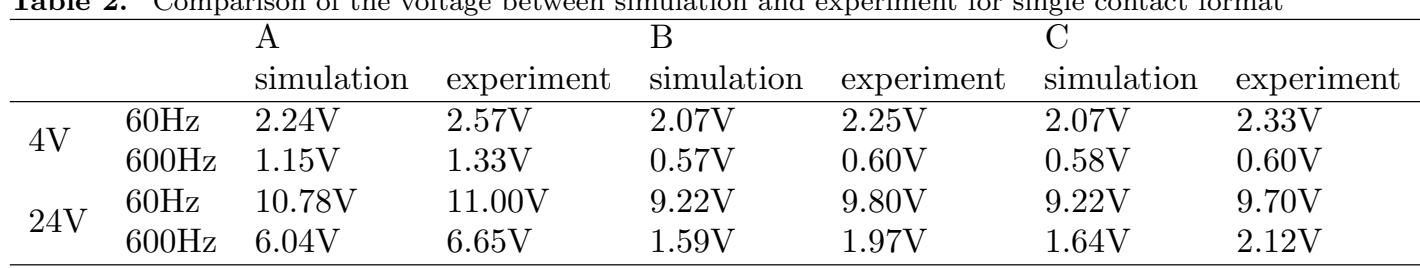

For this single contact format, the transmission is taken at three different positions as indicated in fig.3(b). Position A and B are situated in the corner of the device, one next to the contact and the other away. $\mathrm{C}$ is situated in the centre.

When there is no electric field applied, the liquid crystal in the device is aligned with the rubbing direction, i.e. nearly parallel to the surface, with a small pretilt angle of $2^{\circ}$. Three transmission spectra at position $\mathrm{A}, \mathrm{B}$ and $\mathrm{C}$ are taken under this condition, as shown in fig.8(a).

The shift in wavelength indicates that the liquid crystal layer thickness $\mathrm{d}$ of the cell is not entirely homogeneous. By adjusting the thickness in simulation, the obtained transmission can match with the experimental results, as an example the matching for position A is shown in fig.8(b), with thickness equals to $25.28 \mu \mathrm{m}$. The final thickness used in the simulation is the average value of position $\mathrm{A}, \mathrm{B}$ and $\mathrm{C}$, which equals 25.11 $\mu \mathrm{m}$.

When the sinusoidal wave is applied to the device, the transmission spectra shift according to the reorientation of the LC. The experimental transmission is obtained with a compact halogen light source providing back light and a spectrometer to collect the transmitted light. By adjusting the voltage in the transmission simulation, the spectra obtained are matched with the experimental results, as shown in fig. 8(b). This voltage is used to compare with the voltage simulated from the two coupled differential equations. Both results are shown in table 2. The simulation and experimental results correspond quite well. Slight differences however can be seen. We believe that this is mainly due to the non-uniform thickness of the liquid crystal layer in the real device. Slight changes in thickness can have a strong influence on the optical retardation.

In general, the simulation and the experimental results have a relatively good match, indicating the reliability of the simulation method to predict the homogeneity of a large-sized liquid crystal device. 
Table 3. Power consumption simulated for dashed line contact format

\begin{tabular}{lllll}
\hline voltage format & $4 \mathrm{~V} 60 \mathrm{~Hz}$ & $4 \mathrm{~V} 600 \mathrm{~Hz}$ & $24 \mathrm{~V} 60 \mathrm{~Hz}$ & $24 \mathrm{~V} 600 \mathrm{~Hz}$ \\
\hline power consumption $(\mathrm{W})$ & $0.0188+\mathrm{j} 0.0490$ & $0.1175+\mathrm{j} 0.0524$ & $0.9072+\mathrm{j} 1.9884$ & $4.6864+\mathrm{j} 1.2868$ \\
\hline ideal power consumption $(\mathrm{W})$ & $\mathrm{j} 0.0622$ & $\mathrm{j} 0.622$ & $\mathrm{j} 2.2393$ & $\mathrm{j} 22.393$ \\
\hline
\end{tabular}

\subsection{Contact format: dashed line contact}

It is clear that a single contact in one of the corners of the device is not perfect and may lead to some non-uniformity in the switching of the LC. Thus comes the dashed line contact scheme, wherein the contacts are much more spread out and more homogeneous behaviour of the device is expected. For the dashed line model, each contact has similar format as in the single contact case. There are six contacts each side with pitch $230 \mathrm{~mm}$, and the first and last contact have a distance equal to $20 \mathrm{~mm}$ to the top or bottom edge, seen in fig.3(c). The applied voltage has the same properties as in the previous case.

With a driving voltage of $4 \mathrm{~V}$ and a frequency of $60 \mathrm{~Hz}$, the voltage distribution is shown in fig.9(a), with maximum voltage equals to $3.75 \mathrm{~V}$ and a minimum voltage of $3.71 \mathrm{~V}$, corresponding with a $1 \%$ voltage drop between the edge of the cell and the central part, compared with the applied $4 \mathrm{~V}$. There is an obvious improvement in the voltage distribution as well as an improvement of the uniformity in comparison with the single contact case. As there are more contacts spread over the busbar regions, the RC effect reduces compared to the single contact scheme. Note that the voltage distribution varies more when close to the different contacts, but this non-uniformity fades out when moving away from the contact region. About 10 to $20 \mathrm{~cm}$ away from the busbar region, the voltage distribution is quasi uniform.

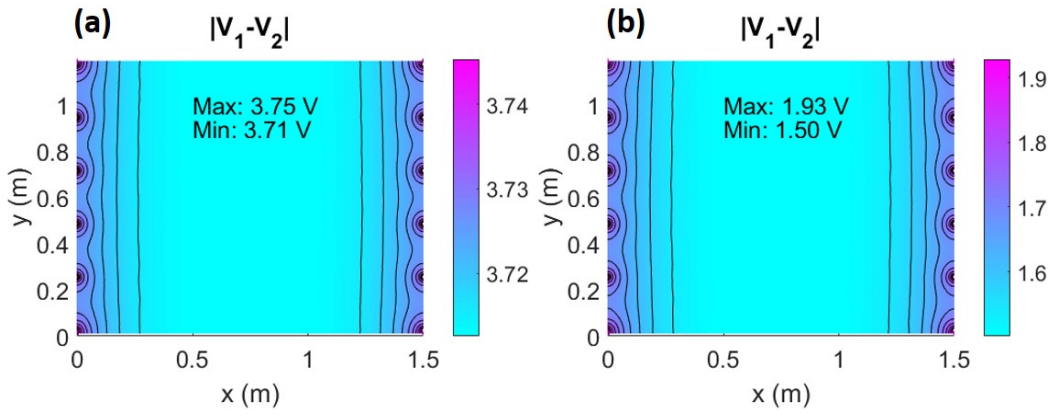

Figure 9. Simulation results for applied sinusoidal signal with voltage $4 \mathrm{~V}$. (a)Voltage distribution with applied frequency $60 \mathrm{~Hz}$. (b)Voltage distribution with applied frequency $600 \mathrm{~Hz}$.

Similar behaviour for the other driving frequency and voltage can be observed for the dashed line contact scheme as for the single contact scheme, as shown in fig.9(b) and fig.10. Overall, the dashed line contact scheme results in improved uniformity and higher overall voltage over the device when compared with the single contact scheme. .7 .

The power consumption for this contact format is shown in table .3 using formula

To verify the simulation results for the dashed line contact format, experiments have also been undertaken on the fabricated device. For this kind of contact format, positions $\mathrm{A}$ and $\mathrm{B}$, as shown in fig.3, are equivalent because of symmetry. Still, measurements on position B have also been taken as comparison. The extracted voltages from the experiments and the simulation results are shown in table 4. The experi- 

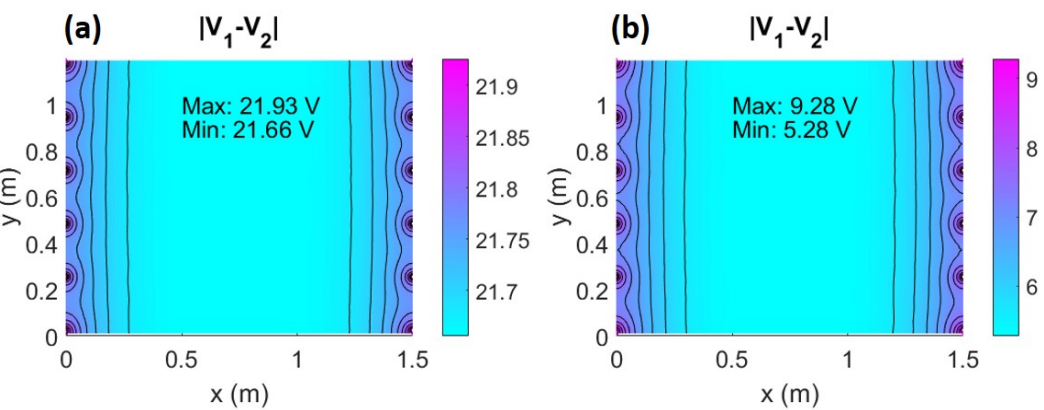

Figure 10. Simulation results for applied sinusoidal signal with voltage $24 \mathrm{~V}$. (a)Voltage distribution with applied frequency $60 \mathrm{~Hz}$. (b)Voltage distribution with applied frequency $600 \mathrm{~Hz}$.

mental and simulation results match quite well except for the voltage of $24 \mathrm{~V}$ and frequency $600 \mathrm{~Hz}$. In principle with more contact dots, the voltage value on the LC should be higher. However, when comparing the experimental value between table 2 and 4 of position A under voltage supply $24 \mathrm{~V} 600 \mathrm{~Hz}$, the dashed line contact format has a lower value, which makes us conclude that probably some error occurred in the measurements. The simulation and experimental results have some minor differences, since the cell thickness is not entirely the same everywhere as explained above, and the large scale makes it easier that there are some deviations between the simulation and fabricated device, the differences can be considered reasonable.

Table 4. Comparison of the voltage between simulation and experiment for dashed line contact format

\begin{tabular}{llllllll}
\hline & & A & \multicolumn{3}{c}{} & B & \\
& & simulation & experiment & simulation & experiment & simulation & experiment \\
\hline \multirow{2}{*}{$4 \mathrm{~V}$} & $60 \mathrm{~Hz}$ & $3.73 \mathrm{~V}$ & $3.75 \mathrm{~V}$ & $3.73 \mathrm{~V}$ & $3.73 \mathrm{~V}$ & $3.71 \mathrm{~V}$ & $3.64 \mathrm{~V}$ \\
& $600 \mathrm{~Hz}$ & $1.72 \mathrm{~V}$ & $1.98 \mathrm{~V}$ & $1.73 \mathrm{~V}$ & $1.94 \mathrm{~V}$ & $1.50 \mathrm{~V}$ & $1.72 \mathrm{~V}$ \\
$24 \mathrm{~V}$ & $60 \mathrm{~Hz}$ & $21.79 \mathrm{~V}$ & $22.00 \mathrm{~V}$ & $21.79 \mathrm{~V}$ & $22.00 \mathrm{~V}$ & $21.66 \mathrm{~V}$ & $21 \mathrm{~V}$ \\
& $600 \mathrm{~Hz}$ & $7.53 \mathrm{~V}$ & $4.24 \mathrm{~V}$ & $7.55 \mathrm{~V}$ & $4.1 \mathrm{~V}$ & $5.23 \mathrm{~V}$ & $3.35 \mathrm{~V}$ \\
\hline
\end{tabular}

\section{Time domain simulation and experiment}

The simulations discussed in the previous sections are based on the coupled differential equations 1 and 2 , where the input is a sinusoidal wave expressed with its root mean square voltage value and frequency. The calculations are conducted in the frequency domain. It is also possible to conduct the simulation in the time domain and define the specific waveform. In this case, the equations should be rewritten as:

$$
\begin{aligned}
& \nabla_{\perp}^{2} V_{1}=\frac{\varepsilon_{\mathrm{eff}}}{\sigma_{1} d_{1} d} \frac{\partial}{\partial t}\left(V_{1}-V_{2}\right) \\
& \nabla_{\perp}^{2} V_{2}=\frac{\varepsilon_{\mathrm{eff}}}{\sigma_{2} d_{2} d} \frac{\partial}{\partial t}\left(V_{2}-V_{1}\right)
\end{aligned}
$$

The single contact format structure is used for this time domain simulation and the applied voltages are chosen to be square waves which have peak voltages of $4 \mathrm{~V}$ and $24 \mathrm{~V}$ with frequencies of $60 \mathrm{~Hz}$ and $600 \mathrm{~Hz}$, respectively. The reason why also square 

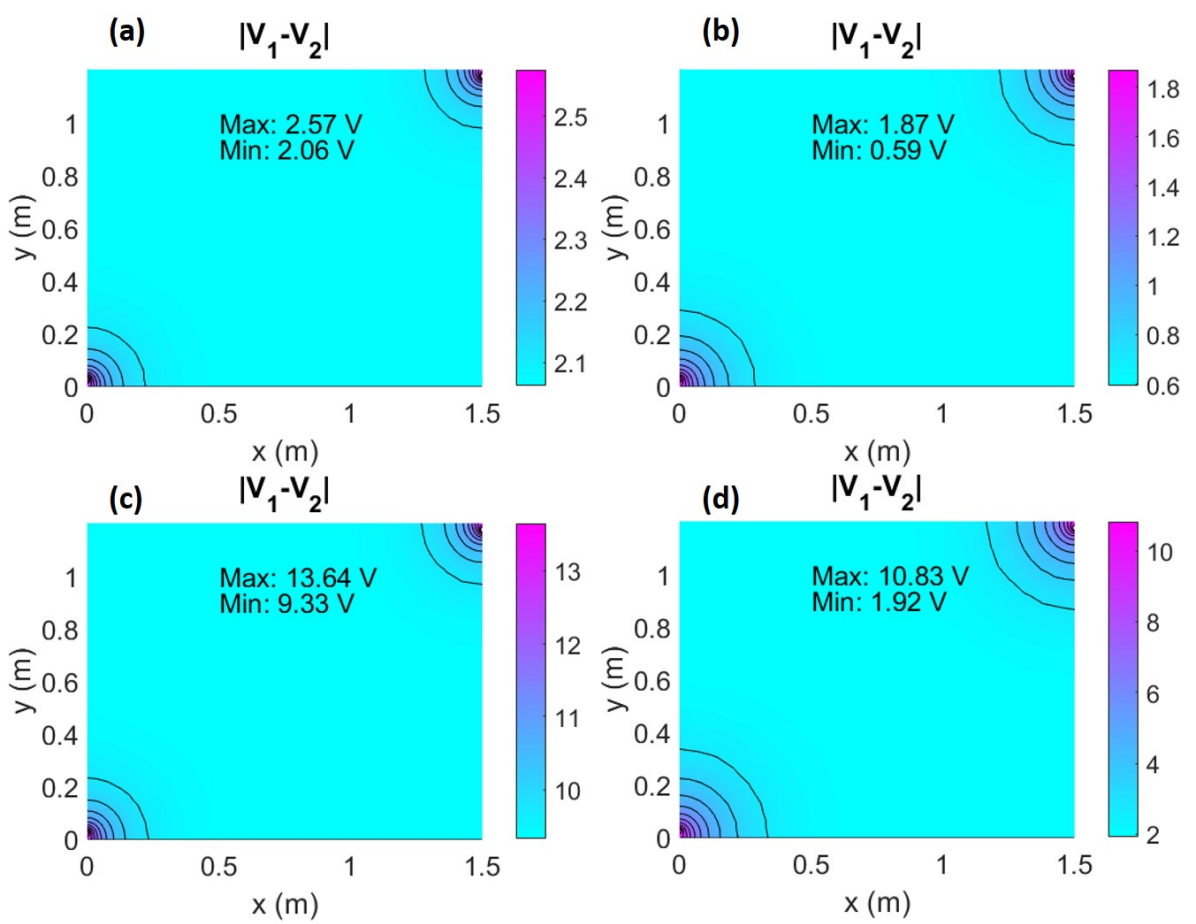

Figure 11. Simulated voltage distribution in the time domain with an applied square wave. (a) $4 \mathrm{~V}$ and $60 \mathrm{~Hz}$. (b) $4 \mathrm{~V}$ and $600 \mathrm{~Hz}$. (c) $24 \mathrm{~V}$ and $60 \mathrm{~Hz}$. (d) $24 \mathrm{~V}$ and $600 \mathrm{~Hz}$.

waves are simulated is the fact that an electronic driver that generates square waves is much easier to implement than a driver for sinusoidal waves [33]. The signal sent to the $\mathrm{LC}$ device is set as a series of six identical square waves. As the calculation is processed in the time domain, the rms voltage is calculated as the square root of the mean of the squared signal, expressed by the following equation: $V_{r m s}=\sqrt{\int_{0}^{T} \frac{1}{T}\left(V_{1}(t)-V_{2}(t)\right)^{2} d t}$ over one period. The resulting rms voltage distribution over the LC device is shown in fig.11.

From this time domain simulation, the homogeneity of the large-sized cell can also be predicted. The voltage drops between the edge and the centre of the cell are calculated to be $12.81 \%, 31.95 \%, 17.97 \%$ and $37.11 \%$ corresponding with fig.11(a), (b), (c) and (d). The similarity in voltage distribution results between the frequency and the time domain is observed due to the similar input voltage parameter.

As previously explained, the interplay between the resistance of the transparent electrodes and the capacitance of the liquid crystal layer causes the RC-effect under $\mathrm{AC}$ voltages. Signals with higher frequency will drop faster than signals with a low frequency. This phenomenon can be easily observed in the square wave case. Since the voltage signal on the transparent conductor consists of several frequency components, the signal will be distorted and will deviate from the original square wave. The temporal evolution of the voltage signal can be extracted at a selected position and compared with the input signal. In the experiment, an oscilloscope is used to record the applied signal to the cell and the actual signal on the ITO layer of the cell at the position of the contact area. The device is fabricated with single contact format, and four types of square waves are applied, with peak voltage amplitudes of $4 \mathrm{~V}$ and $24 \mathrm{~V}$ and frequencies of $60 \mathrm{~Hz}$ and $600 \mathrm{~Hz}$. The comparison between the input signal 
format and the signal format extracted from the contact area for both simulation and experiment is shown in fig. 12 and 13.

(a)

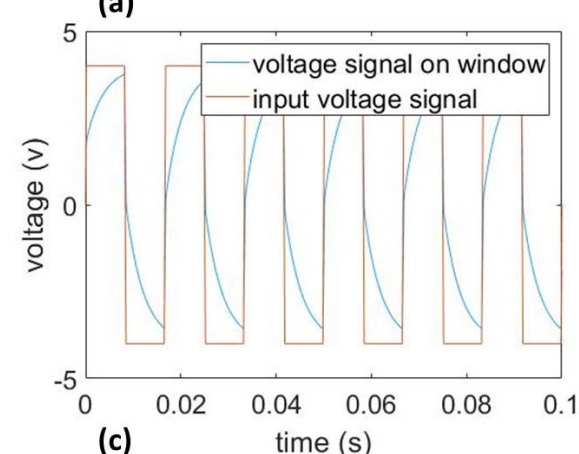

(b)
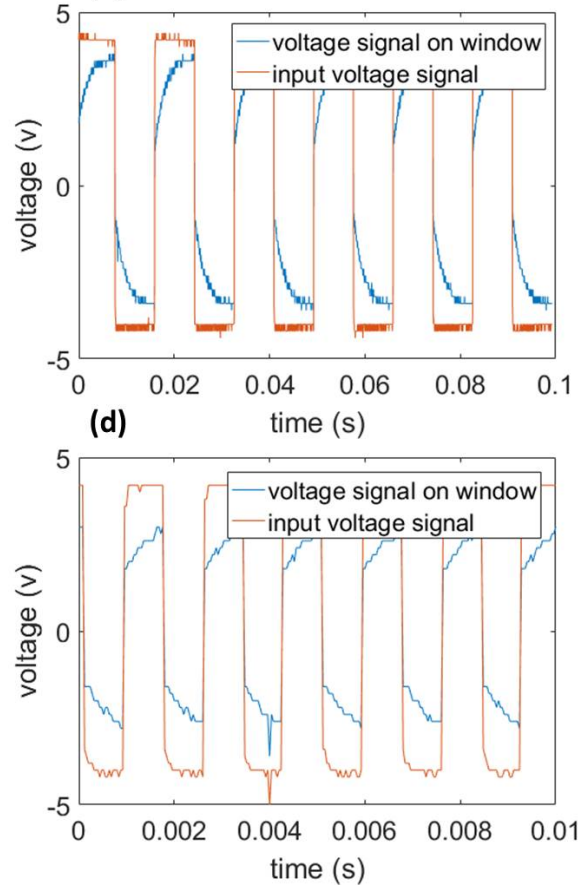

Figure 12. Comparison of voltage signals when a square wave voltage is applied. (a) \& (c) Simulation; (b) \& (d) Experiment. (a) \& (b) $4 \mathrm{~V}$ and $60 \mathrm{~Hz}$. (c) \& (d) $4 \mathrm{~V}$ and $600 \mathrm{~Hz}$.

From the figure, the RC-effect can be seen intuitively from the drop of the voltage signal format, and the higher the applied frequency, the stronger the effect is. For the input square wave signal with $4 \mathrm{~V}$ and $60 \mathrm{~Hz}$, the effective voltage on the contact area is $2.57 \mathrm{~V}$, indicating a drop of $35.75 \%$ compared with input; while for the $600 \mathrm{~Hz}$ case, the effective voltage decreases to $1.87 \mathrm{~V}$, corresponding with a drop of $53.52 \%$. The similar phenomenon can be observed with the $24 \mathrm{~V}$ input case, with the effective voltages drop to $13.64 \mathrm{~V}$ and $10.83 \mathrm{~V}$ for the $60 \mathrm{~Hz}$ and $600 \mathrm{~Hz}$ signal respectively.

\section{Conclusion}

In this work, a model is built based on coupled partial differential equations to simulate the behaviour for large-sized liquid crystal devices. Different contact formats and applied voltages are used for the analysis of the behaviour. A LC device is fabricated with E7 as the functioning LC and experiments are conducted to compare with the simulation results. The match between the simulation and experiment verifies the reliability of this model, which could also be used in the future to predict the behaviour for other kinds of large-sized liquid crystal device. Our model offers an indispensable tool for selecting an appropriate sheet resistance and voltage signal that offers an acceptable value of the optical uniformity of the device with an acceptable transmission of the transparent conductor. This model works in a way that LC behaviour from across a large surface would all be predicted. Different parameters corresponding with specific properties are considered and could be flexibly adjusted according to certain 

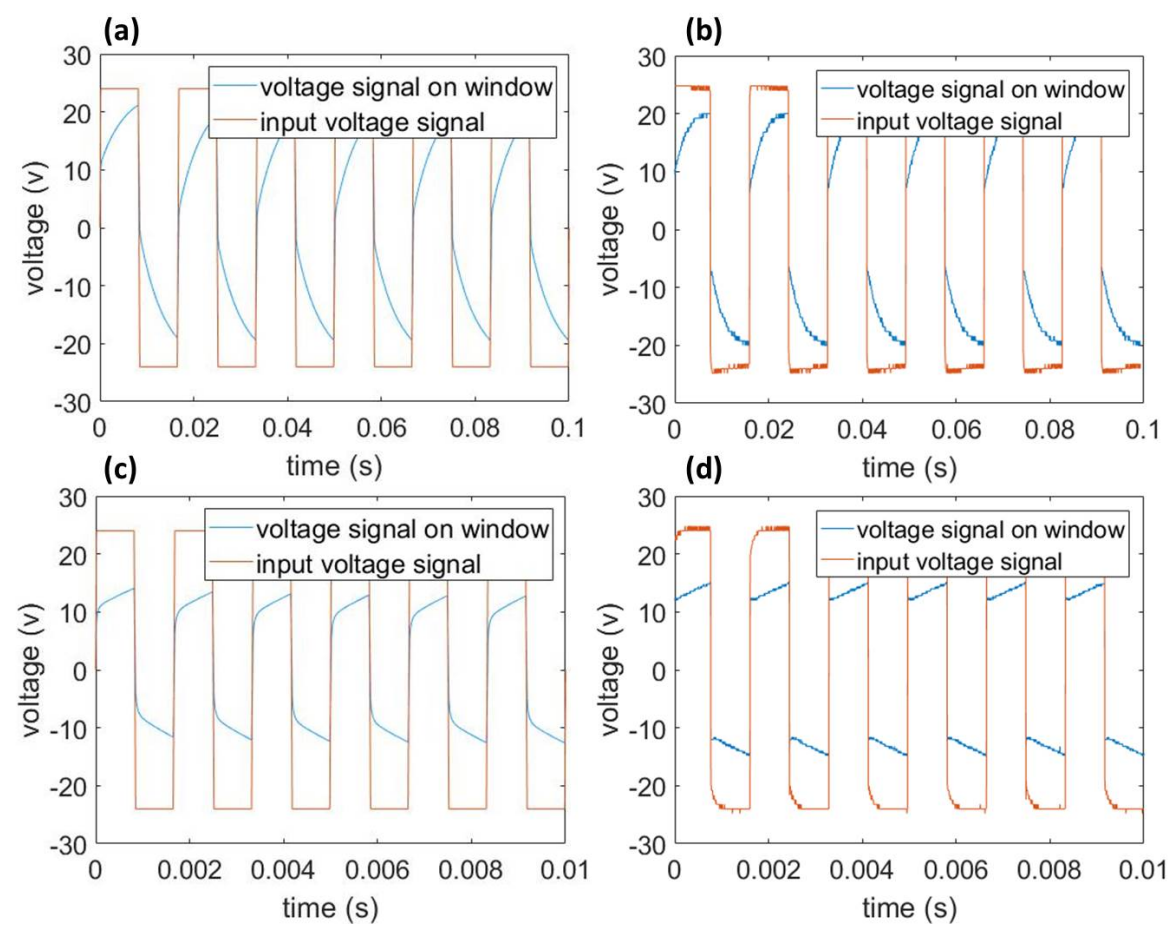

Figure 13. Comparison of voltage signals when a square wave voltage is applied. (a) \& (c) Simulation; (b) \& (d) Experiment. (a) \& (b) $24 \mathrm{~V}$ and $60 \mathrm{~Hz}$. (c) \& (d) $24 \mathrm{~V}$ and $600 \mathrm{~Hz}$.

applications. The calculation of the power consumption of the device shows that in general, the sandwiched LC cell does not act as a pure capacitor. Resistive effects from the finite sheet conductivity also need to be taken into account. Lastly, it is clear from the simulations that sinusoidal waves are the best way to drive the device as the higher frequency components in a square wave are much stronger attenuated compared to the fundamental frequency component.

\section{References}

[1] Baetens R, Jelle BP, Gustavsen A. Properties, requirements and possibilities of smart windows for dynamic daylight and solar energy control in buildings: A state-of-the-art review. Sol Energy Mater Sol Cells. 2010;94(2):87-105.

[2] Wang J, Zhang L, Yu L, et al. A bi-functional device for self-powered electrochromic window and self-rechargeable transparent battery applications. Nat Commun. 2014;5:4921.

[3] Piccolo A. Thermal performance of an electrochromic smart window tested in an environmental test cell. Energy Build. 2010;42(9):1409-1417.

[4] Chen CW, Brigeman AN, Ho TJ, et al. Normally transparent smart window based on electrically induced instability in dielectrically negative cholesteric liquid crystal. Opt Mater Express. 2018;8(3):691-697.

[5] Granqvist CG. Oxide electrochromics: An introduction to devices and materials. Sol Energy Mater Sol Cells. 2012;99:1-13.

[6] Feng W, Zou L, Gao G, et al. Gasochromic smart window: optical and thermal properties, energy simulation and feasibility analysis. Sol Energy Mater Sol Cells. 2016;144:316-323.

[7] Costanzo V, Evola G, Marletta L. Thermal and visual performance of real and theoretical thermochromic glazing solutions for office buildings. Sol Energy Mater Sol Cells. 2016; 
149:110-120.

[8] Azens A, Granqvist C. Electrochromic smart windows: energy efficiency and device aspects. J Solid State Electrochem. 2003;7(2):64-68.

[9] Gardiner D, Morris S, Coles H. High-efficiency multistable switchable glazing using smectic a liquid crystals. Sol Energy Mater Sol Cells. 2009;93(3):301-306.

[10] Ghosh A, Norton B, Duffy A. Measured overall heat transfer coefficient of a suspended particle device switchable glazing. Appl Energy. 2015;159:362-369.

[11] Lampert C. Large-area smart glass and integrated photovoltaics. Sol Energy Mater Sol Cells. 2003;76(4):489-499.

[12] Macrelli G. Optical characterization of commercial large area liquid crystal devices. Sol Energy Mater Sol Cells. 1995;39(2):123-131.

[13] Lampert C. Smart windows switch on the light. IEEE Circuits Devices Mag. 1992;8(2):1926.

[14] Ke Y, Zhou C, Zhou Y, et al. Emerging thermal-responsive materials and integrated techniques targeting the energy-efficient smart window application. Adv Funct Mater. 2018;28(22):1800113.

[15] Ghosh A, Norton B, Mallick TK. Daylight characteristics of a polymer dispersed liquid crystal switchable glazing. Sol Energy Mater Sol Cells. 2018;174:572-576.

[16] Murray J, Ma D, Munday JN. Electrically controllable light trapping for self-powered switchable solar windows. ACS Photonics. 2016;4(1):1-7.

[17] Khaligh HH, Liew K, Han Y, et al. Silver nanowire transparent electrodes for liquid crystal-based smart windows. Sol Energy Mater Sol Cells. 2015;132:337-341.

[18] Sun H, Xie Z, Ju C, et al. Dye-doped electrically smart windows based on polymerstabilized liquid crystal. Polymers. 2019;11(4):694.

[19] Kim JH, Huh JW, Oh SW, et al. Bistable switching between homeotropic and focal-conic states in an ion-doped chiral nematic liquid crystal cell. Opt Express. 2017;25(23):29180 29188.

[20] Raj D. Dichroic display technology potentials and limitations. Mater Chem Phys. 1996; 43(3):204-211.

[21] Gordon RG. Criteria for choosing transparent conductors. MRS Bull. 2000;25(8):52-57.

[22] Kim IC, Kim TH, Lee SH, et al. Extremely foldable and highly transparent nanofiberbased electrodes for liquid crystal smart devices. Sci Rep. 2018;8(1):11517.

[23] Wassei JK, Kaner RB. Graphene, a promising transparent conductor. Mater Today. 2010; 13(3):52-59.

[24] Neyts K, Beunis F. Ion transport in liquid crystals. Handb Liq Cryst. 2014;:1-26.

[25] Beunis F, Strubbe F, Marescaux M, et al. Dynamics of charge transport in planar devices. Phys Rev E. 2008;78(1):011502.

[26] Beeckman J, Nys I, Willekens O, et al. Optimization of liquid crystal devices based on weakly conductive layers for lensing and beam steering. J Appl Phys. 2017;121(2):023106.

[27] Neyts K, Real A, Marescaux M, et al. Conductor grid optimization for luminance loss reduction in organic light emitting diodes. J Appl Phys. 2008;103(9):093113.

[28] Tang H, Jiang Y, Tang CW, et al. Grid optimization of large-area oled lighting panel electrodes. J Disp Technol. 2016;12(6):605-609.

[29] Cheknane A, Benyoucef B, Charles JP, et al. Minimization of the effect of the collecting grid in a solar cell based silicon. Sol Energy Mater Sol Cells. 2005;87(1-4):557-565.

[30] Yeh P, Gu C. Optics of liquid crystal displays. Vol. 67. [place unknown]: John Wiley \& Sons; 2009.

[31] Beeckman J, Neyts K, Hutsebaut X, et al. Simulation of 2-d lateral light propagation in nematic-liquid-crystal cells with tilted molecules and nonlinear reorientational effect. Opt Quantum Electron. 2005;37(1-3):95-106.

[32] Li J, Wen CH, Gauza S, et al. Refractive indices of liquid crystals for display applications. J Disp Technol. 2005;1(1):51.

[33] Minaei S, Yuce E. A simple schmitt trigger circuit with grounded passive elements and its application to square/triangular wave generator. Circuits Syst Signal Process. 2012; 
31(3):877-888 